\title{
Atomic Oxygen Sensing Using a Quartz Crystal Microbalance with a Polymer Thin Film Prepared by RF Sputtering
}

\author{
Hiroyuki Matsumoto*, Mikihiko Matsuoka, \\ Satoru Iwamori ${ }^{1}$ and Kazutoshi Noda ${ }^{2}$ \\ Corporate Advanced Technology Center, Iwasaki Electric Co., Ltd., \\ 1-20 Fujimicho, Gyoda 361-0021, Japan \\ ${ }^{1}$ Graduate School of Natural Science and Technology, Kanazawa University, \\ Kakuma-machi, Kanazawa 920-1192, Japan \\ ${ }^{2}$ Institute for Environmental Management Technology, National Institute of Advanced Industrial \\ Science and Technology (AIST), Onogawa, Tsukuba 305-8569, Japan
}

(Received February 27, 2009; accepted July 6, 2009)

Key words: atomic oxygen, quartz crystal microbalance, polyimide, RF sputtering

Polymer thin films, sputtered using a polyimide target on a quartz crystal, were investigated to determine their suitability for application in active oxygen sensors. Active oxygen generated under an inductively coupled plasma (ICP), particularly atomic oxygen radicals with a flux of $6.93 \times 10^{13}$ atoms $/ \mathrm{cm}^{2} / \mathrm{s}$, was successfully detected from the frequency shift using a polymer-coated quartz crystal microbalance (QCM). To investigate the reaction mechanism between the polymer surface and atomic oxygen, chemical bonding was evaluated by electron spectroscopy for chemical analysis (ESCA) and surface morphology by atomic force microscopy (AFM). We also compared sputtercoated polymers with spin-coated polymers in terms of their sensing characteristics. We found that a sputter-coated polymer QCM has a great potential for atomic oxygen sensing.

\section{Introduction}

Active oxygen, generated by ultraviolet lamps, ozonizers, and discharge plasma, plays an important role in several industrial processes, including surface cleaning, surface modification, sterilization, and oxidation. With the current need to reduce greenhouse gas emissions (e.g., fluorocarbons used in semiconductor manufacturing processes), the use of active oxygen is attracting more attention. In particular, atomic oxygen has an extremely strong oxidative ability and could therefore be applied to the above processes. These processes, however, demand precise reproducibility from the viewpoint of attaining a high production yield. Therefore, being able to accurately measure the amount of atomic oxygen is critical to the precise control of these processes. ${ }^{*}$ Corresponding author: e-mail: matsumoto-hiroyuki@eye.co.jp 
Such measurement requires costly specialized equipment incorporating a laser optical system $^{(1)}$ or a vacuum ultraviolet light source, ${ }^{(2,3)}$ such that a reasonably priced and simple method of real-time sensing is not currently available to the best of our knowledge.

A quartz crystal microbalance (QCM) is a sensor device that is capable of the nanogram-order measurement of changes in mass on the surface of a quartz crystal by observing shifts in resonant frequency.(4) Hence, this method can have different gassensing applications by selecting the appropriate electrode material to be formed on the quartz crystal as the detection layer. ${ }^{(5)}$ In our previous study, we verified the atomic oxygen sensing and accuracy of carbon-coated and silver-coated QCMs. ${ }^{(6)}$ Although the silver-coated QCM is highly sensitive, frequency changes rapidly over several seconds and without any linearity as a result of the marked surface oxidation by atomic oxygen. Therefore, a silver-coated QCM cannot provide stable sensing in industrial processes. On the other hand, the carbon-coated QCM showed stable detection properties. Carbon thin films, however, generally applied by a flash deposition method based on the resistive heating of a pair of carbon rods, suffer from a serious drawback; it is difficult to obtain thick films and reproduce film thickness owing to restrictions imposed by the deposition principle. Clearly, a reproducible mass is indispensable from the viewpoint of practical use. This is disadvantageous when carbon thin films are applied to processes that require atomic oxygen sensing over a longer duration.

Polymer films such as polyimide (PI), polyethylene terephthalate (PET), polyvinyl fluoride (PVF), and polytetrafluoroethylene (PTFE) exhibit relatively low reaction efficiencies for atomic oxygen over an extended duration. ${ }^{(7)}$ To fabricate a durable atomic oxygen sensor, we first developed a polymer-coated QCM that was prepared by RF sputtering with a polyimide target, because this method can readily produce thin films with excellent thickness controllability and reproducibility. In this paper, we investigate the atomic oxygen sensing properties of this QCM. In particular, the long-term (over $30 \mathrm{~min}$ ) sensing property was verified to determine whether the QCM could be used for practical applications. Moreover, we considered the effect of the atomic oxygen sensing mechanism on a polymer-coated QCM by evaluating surface chemical bonding states and morphologies.

\section{Experimental Procedure}

\subsection{Preparation of polymer-coated $Q C M$}

The QCM is a sensor device that supports the nanogram-order measurement of changes in mass on the surface of a quartz crystal by observing frequency shifts. In this study, we investigated the use of the QCM technique for sensing active oxygen species, particularly atomic oxygen. A polymer thin film was formed as the atomic oxygen sensing layer on a commercially available AT-cut quartz crystal (with a resonant frequency of $6 \mathrm{MHz}$, a diameter of $14 \mathrm{~mm}$, and gold electrodes on both sides) by RF sputtering with a polyimide target (Upilex-S, Ubekousan).

The coating was applied as follows. A quartz crystal plate was mounted in the sputtering equipment (SBR-1104E, ULVAC). After evacuating the equipment to less 
than $6.7 \times 10^{-3} \mathrm{~Pa}$, argon (Ar) gas was introduced at $5.3 \times 10^{-1} \mathrm{~Pa}$. An RF $(13.56 \mathrm{MHz})$ power of $150 \mathrm{~W}$ was applied between the target and the stainless steel substrate holder, and then polymer coating onto the quartz plate was carried out. (The sputter-coated polymer films are described in greater detail in ref. 8.) The thin film was formed on one side, the area being ca. $8 \mathrm{~mm}$ in diameter and ca. $75 \mathrm{~nm}$ thick, which was determined by measuring the heights between the film and the noncoated area with a contact-type roughness gauge. We can assume that the film has no complete polyimide structure because of the decomposition caused by sputtering with argon ions.

For comparison, a polyimide amide acid (Semicofine SP-341, Toray Industries, Inc.) was prepared and coated on the above-mentioned quartz crystal using a spin coater (K-359 $\mathrm{S}-1$, Kyowariken). The amide acid was diluted to $1 / 4$ of the original concentration in $N$-methyl-2-pyrrolidine, and then $10 \mu \mathrm{l}$ of the obtained solution was dropped onto the quartz crystal mounted in the spin coater. The spin-coating speed was set to $3000 \mathrm{rpm}$ for $100 \mathrm{~s}$, and the film was cured to dehydrate and form the polyimide structure at $140^{\circ} \mathrm{C}$ for $30 \mathrm{~min}, 200^{\circ} \mathrm{C}$ for $30 \mathrm{~min}$, and then $300^{\circ} \mathrm{C}$ for $1 \mathrm{~h}$. The film coating was formed over the entire quartz crystal, and the obtained film thickness was ca. $150 \mathrm{~nm}$.

\subsection{Experimental setup and procedure for atomic oxygen sensing}

Figure 1 shows the setup of the atomic oxygen irradiation chamber used in this study. The equipment features an inductively coupled plasma (ICP) source in the upper part of the vacuum chamber. This acts as the atomic oxygen source. The vacuum chamber was evacuated to less than $7 \times 10^{-1} \mathrm{~Pa}$ using a rotary pumping system, and then oxygen gas ( $5 \mathrm{~N}$ purity) was introduced at a constant flow rate of $0.05 \mathrm{SLM}$ (standard liters/min) by mass flow (MODEL 3660, KOFLOC) at a pressure of $25 \mathrm{~Pa}$. After a $30 \mathrm{~s}$ pause, it was confirmed that the pressure was stable, and then an RF $(13.56 \mathrm{MHz})$ power of $200 \mathrm{~W}$ was supplied to the antenna through the matching network such that inductively coupled oxygen plasma was generated within the ICP source. Early in this study, we assumed that there are many active oxygen species (e.g., oxygen ions, excited molecules, and ozone) in the plasma and vacuum chamber regions, although a subsequent spectroscopic study showed that atomic oxygen radicals $\left(\mathrm{O}^{*}:{ }^{1} \mathrm{D} \rightarrow{ }^{3} \mathrm{P}\right.$ state $)$ are the predominant form of active oxygen in the irradiation area. These atomic oxygen radicals are assumed to flow downwards, drawn by the flow of evacuation. The QCM sensor was inserted into the sensor head and then connected to the oscillation circuit at a position $35 \mathrm{~mm}$ below the ICP source and on the central axis of the equipment. Frequency shifts during plasma generation were monitored every $30 \mathrm{~s}$ by a frequency counter (SC-7205, Iwatsu), and the results were recorded.

\section{Results and Discussion}

To verify the possibility of atomic oxygen sensing using a polymer-coated QCM, we first investigated the frequency shift of sputter-coated and spin-coated polymer QCMs during irradiation with atomic oxygen. The results are shown in Fig. 2. The total sensing time was set to $10 \mathrm{~min}$, including $30 \mathrm{~s}$ to confirm the sensor stability with only the oxygen gas flow (from 0 to $0.5 \mathrm{~min}$, in Fig. 2). For the sputter-coated QCM, the 


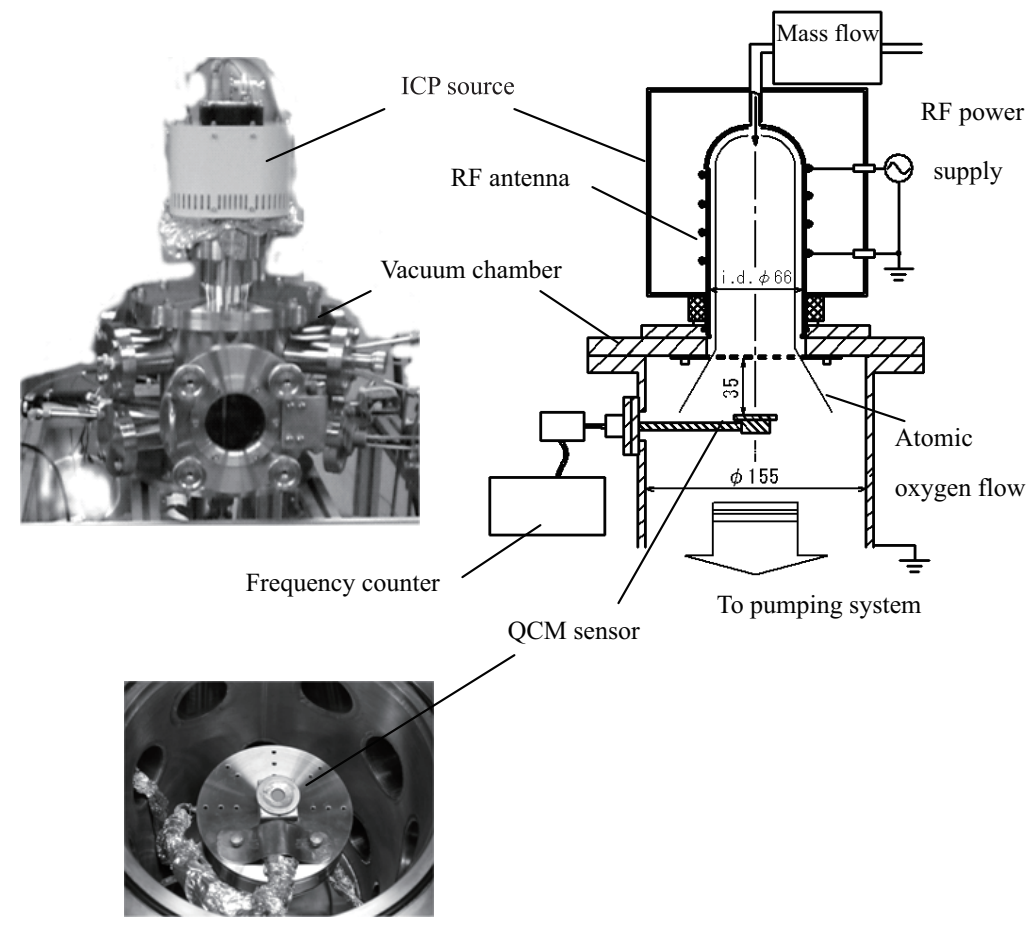

Fig. 1. Experimental setup.

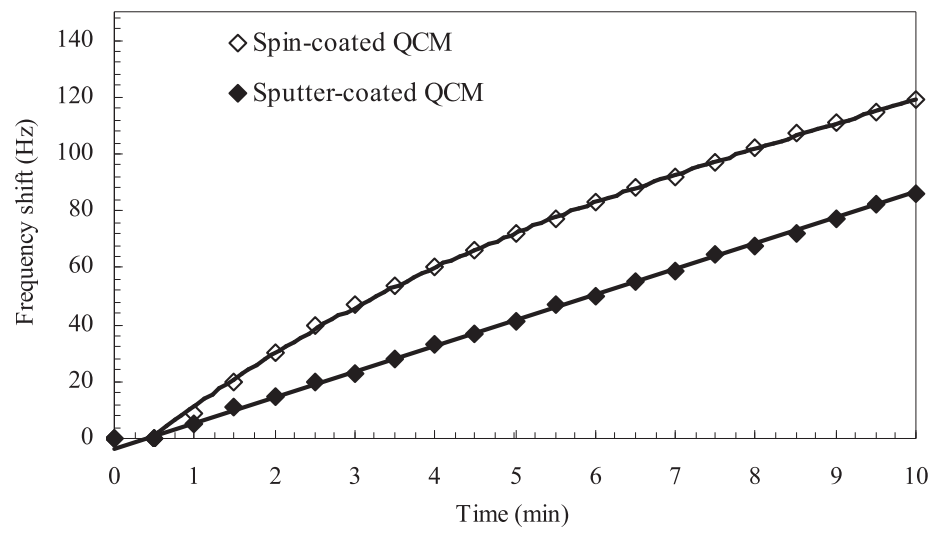

Fig. 2. Frequency shift of polymer-coated QCM. (Atomic oxygen flux: $6.93 \times 10^{13}$ atoms $/ \mathrm{cm}^{2} / \mathrm{s}$ ) 
frequency increased linearly to $86 \mathrm{~Hz}$ for over $9 \mathrm{~min}$. This frequency shift value is ca. four times larger than the result obtained with the carbon-coated QCM $(22 \mathrm{~Hz})$ under the same irradiation conditions that correspond to an atomic oxygen flux of $6.93 \times 10^{13}$ atoms/ $\mathrm{cm}^{2} / \mathrm{s}^{\left({ }^{(9)}\right.}$

This flux number, which is estimated assuming that the carbon is removed by atomic oxygen and then volatile flux (CO) is formed, indicates the lower limit of detection by this QCM, due to variations in frequency shift behavior. As mentioned above, we also evaluated optical emission spectroscopy as a means of qualitatively analyzing atomic oxygen. However, the emission intensity decreases with the distance from the ICP source and is very weak near the QCM sensor. Under these conditions, surface treatments often take a long time in practical industrial applications, i.e., this indicates that an atomic oxygen flux on the order of $10^{13}$ is relatively inefficient for surface treatments. This fact suggests that the QCM enables the detection of small quantities of atomic oxygen with a high resolution.

On the other hand, the spin-coated QCM exhibited a phased frequency shift for 6 min from the beginning of the atomic oxygen irradiation. Then, the frequency increased up to $120 \mathrm{~Hz}$. The total frequency shift of the spin-coated QCM was larger than that of the sputter-coated QCM, and the difference was slightly less than $40 \mathrm{~Hz}$, as shown in Fig. 2.

Iwamori et al. noted that the sputter-coated polymer has a broad FT-IR spectrum, which differs from the bulk polyimide. This suggests that new functional groups were generated in the polymer as a result of RF sputter coating with a polyimide target. ${ }^{(10)}$ Therefore, the reason for this difference in frequency shift behavior between the spinand sputter-coated QCMs is assumed to be the difference in the moieties of the polymer thin films. Namely, this result indicates that the difference in reaction efficiency with atomic oxygen is due to the difference in the molecular structures of the thin films.

As mentioned below, the sputter-coated QCM exhibits stable detection properties over a long duration despite a reduction in its weight. In other words, the difference in the initial film thickness, spin-coated to $150 \mathrm{~nm}$ and sputter-coated to $75 \mathrm{~nm}$, is thought to have no substantial relationship with this result. We also confirmed that the initial thickness of the sputter-coated polymer has no influence on the detection properties.

For the sputter-coated QCM, it is thought that the reaction with atomic oxygen was slightly suppressed owing to the formation of functional groups, such that the frequency shift is reduced. These results indicate that the sputter-coated polymer QCM enables the highly sensitive quantification of atomic oxygen from the linear slope of the frequency shift, and is superior to the conventional carbon-coated QCM.

Next, to clarify the mechanism of atomic oxygen sensing on the polymer-coated QCM, we evaluated the surface chemical bonding state of the sputter-coated polymer QCM using electron spectroscopy for chemical analysis (ESCA) (AXIS Ultra, KRATOS). ESCA measurement was performed in a high vacuum of less than $6 \times 10^{-6}$ $\mathrm{Pa}$, with a monochromatic X-ray source (Al-K $\alpha, 10 \mathrm{kV}, 10 \mathrm{~mA})$. First, a wide scan was performed in the energy range from 1350 to $-5 \mathrm{eV}$ in $1 \mathrm{eV}$ steps.

For the ESCA wide-scan spectra, prominent peaks corresponding to N1s, O1s, and $\mathrm{C} 1 \mathrm{~s}$ were observed. The peak intensity ratio of $\mathrm{O} 1 \mathrm{~s} / \mathrm{C} 1 \mathrm{~s}$ increases from 0.41 (before irradiation) to 0.86 (after 10 min irradiation), as shown in Table 1, which might be caused 
Table 1

$\mathrm{O} 1 \mathrm{~s} / \mathrm{C} 1 \mathrm{~s}$ ratio of sputter-coated polymer before and after irradiation.

\begin{tabular}{lccc}
\hline & $\begin{array}{c}\text { C1s relative } \\
\text { intensity (arb. unit) }\end{array}$ & $\begin{array}{c}\text { O1s relative } \\
\text { intensity (arb. unit) }\end{array}$ & $\begin{array}{c}\text { O1s/C1s } \\
\text { ratio }\end{array}$ \\
\hline Before & 29795 & 12115 & 0.41 \\
After & 21750 & 18791 & 0.86 \\
\hline
\end{tabular}

by carbon removal and surface oxidation under the influence of atomic oxygen.

To analyze the sensing reaction in greater detail, particularly the removal of organic compounds, which leads to polymer weight loss, a narrow scan in $0.1 \mathrm{eV}$ steps with regard to the $\mathrm{C} 1 \mathrm{~s}$ spectra was evaluated. Gaussian curve fitting and peak synthesis were performed for the obtained $\mathrm{C} 1 \mathrm{~s}$ spectra at each binding energy. The results are shown in Table 2 and Fig. 3. Then, the full width at half-maximum (FWHM) of the fitting curve was fixed to $1.4 \mathrm{eV}$ using the method reported by Kinoshita et al. ${ }^{(1)}$ The atomic compositions listed in Table 2 were calculated from the ratio of the area under each curve. A decrease in the BPDA and PDA contents and an increase in those of all the oxidative functional groups $-\mathrm{C}-\mathrm{O}-\mathrm{C}-,-\mathrm{C}=\mathrm{O}-,-\mathrm{C}-\mathrm{O}-\mathrm{N}-$, and $-\mathrm{CO}-\mathrm{OH}-$ were clearly observed. The trends appearing in these results are in good agreement with the report published by Yokota et al., in which the durability of the spin-coated polyimide in the presence of atomic oxygen was clarified. ${ }^{(12)}$

The polymer surface morphologies, both before and after the atomic oxygen sensing, were evaluated using atomic force microscopy (AFM; SPA-300, Seiko Instruments). The scan mode was set to the dynamic force mode (DFM). The scan frequency was 2.0 $\mathrm{Hz}$ and the area was $1000 \mathrm{~nm}$. Figure 4 shows an arbitrary cross-sectional profile of the polymer surface. Note that a polymer thin-film coating was formed on a polished silicon wafer having a nano-order flat surface to eliminate the effect of roughness caused by the substrate surface. We confirmed that the pristine polymer surface has a smooth surface morphology with a root-mean-square (RMS) roughness of ca. $0.17 \mathrm{~nm}$. In contrast, we observed the formation of fine protuberances with a height of $1.4 \mathrm{~nm}$ and an RMS roughness of $0.36 \mathrm{~nm}$ on the irradiated surface.

From a series of the above results, the mechanisms of the reaction between atomic oxygen and polyimide are assumed to be as follows. Functional groups, e.g., phenol compounds, are produced as a result of the absorption of atomic oxygen onto the imide or benzene rings of the polyimide, after which the cleavage of the rings advances as a result of a cycloaddition and/or other reactions (scheme (1), in Fig. 5). After the cleavage of the benzene and imide rings, olefin or aldehyde compounds containing unsaturated carbon are produced (scheme (2)). Carbonyl moieties of these compounds are finally desorbed as volatile fluxes such as $\mathrm{CO}$ or $\mathrm{CO}_{2}$ owing to further reaction with atomic oxygen (scheme (3)).(13) This phenomenon leads to the weight loss of the polymer and consequently to the frequency increase of the QCM, which enables atomic oxygen sensing.

The above assumptions relate to the "bulk-polyimide surface." It is thought, however, that a similar reaction based on these assumptions occurs not only on the bulk polyimide 
Table 2

Synthesis results.

\begin{tabular}{lcccccc}
\hline \multicolumn{7}{c}{ Composition (at.\%) } \\
\hline & PDA & BPDA & $-\mathrm{C}-\mathrm{O}-\mathrm{C}-$ & $-\mathrm{C}=\mathrm{O}-$ & $-\mathrm{C}-\mathrm{O}-\mathrm{N}-$ & $-\mathrm{CO}-\mathrm{OH}-$ \\
& $285.0 \mathrm{eV}$ & $286.0 \mathrm{eV}$ & $286.6 \mathrm{eV}$ & $287.7 \mathrm{eV}$ & $288.9 \mathrm{eV}$ & $289.6 \mathrm{eV}$ \\
\hline Before & 68.7 & 15.9 & 5.8 & 7.1 & 2.3 & 0.3 \\
After & 59.6 & 14.0 & 11.8 & 8.9 & 5.3 & 0.4 \\
\hline
\end{tabular}

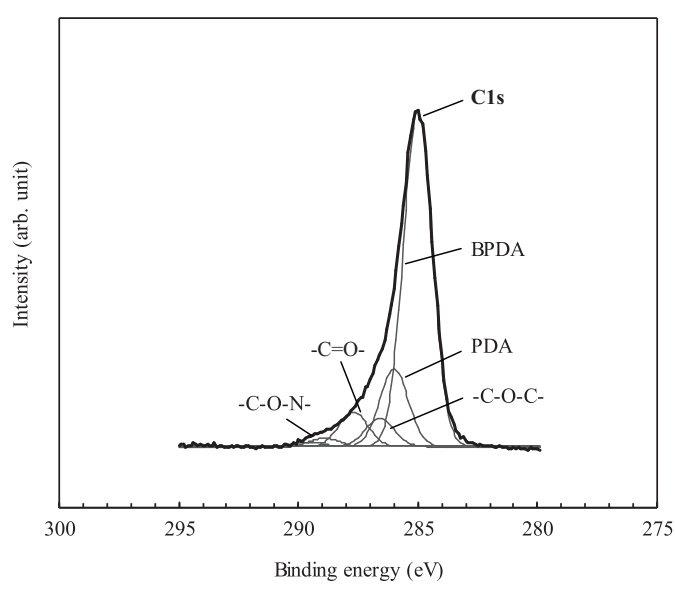

(a)

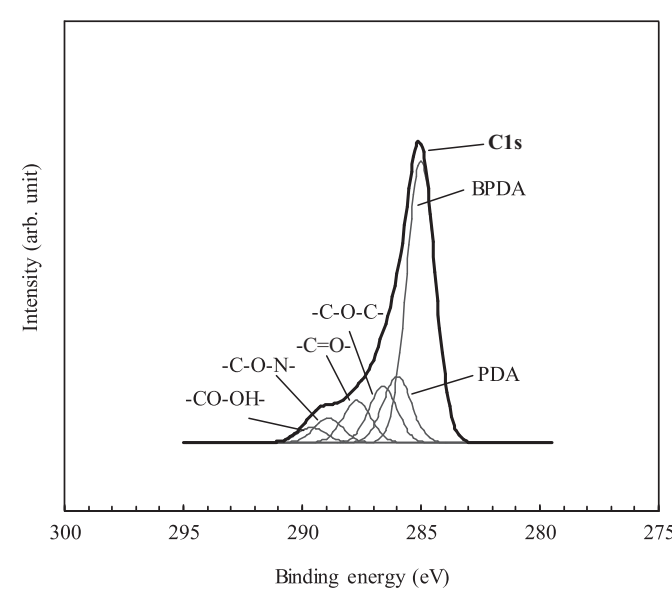

(b)

Fig. 3. Curve fitting for C1s spectra of sputter-coated polymer. (a) Before irradiation. (b) After irradiation.

but also on the sputter-coated polymer surface, because these effects are consistent with the ESCA results, i.e., an increase in the the oxidative functional group content and a decrease in the BPDA/PDA structure content after the atomic oxygen irradiation of the sputter-coated polymer surface. Besides, there is a possibility that new functional groups are formed on the polymer surface, which do not appear on the bulk polyimide, leading to a difference in the reaction efficiency with atomic oxygen, as shown in Fig. 2.

The fine protuberance formation on the irradiated polymer surface, as verified in the AFM image where no flat-etching surface appears, indicates the formation of residual moieties as a result of atomic oxygen reactions in the above-mentioned schemes.

To investigate long-term atomic oxygen sensing, which is the goal of this study, the frequency shift characteristic of the sputter-coated QCM, as measured for more than 30 min, was determined. The experimental conditions were the same as those described above, with the exception of sensing time. Figure 6 shows the frequency shift result. From these results, we find that frequency increases up to $255 \mathrm{~Hz}$ in $35 \mathrm{~min}$. This suggests that the sputter-coated QCM has great potential for atomic oxygen sensing over 
(a)
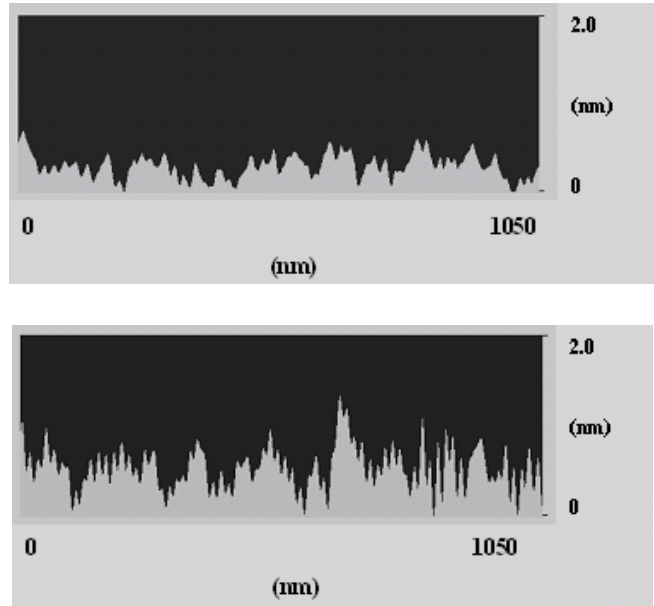

Fig. 4. Arbitrary cross-sectional profile of polymer thin film. (a) Before irradiation. (b) After irradiation.
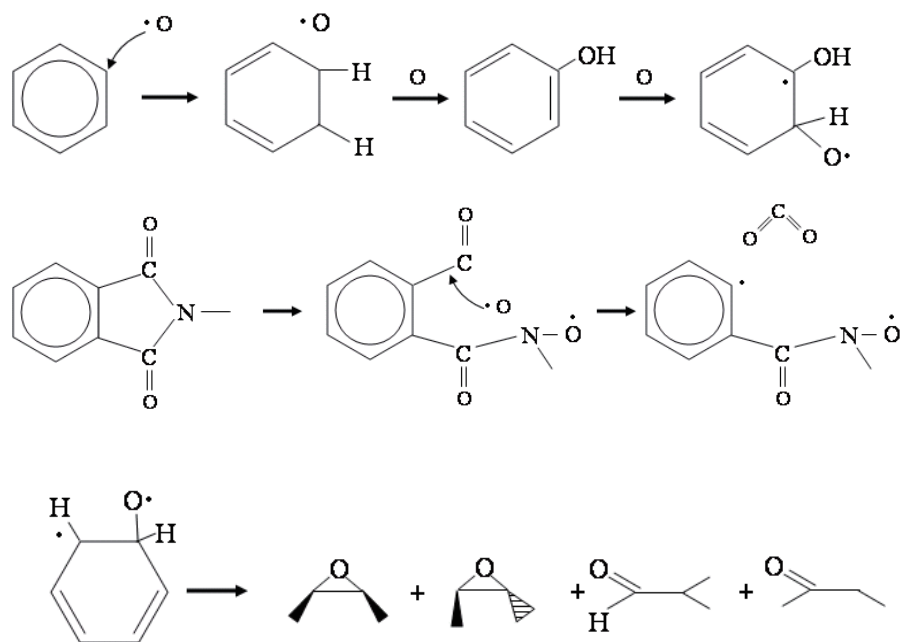

$\mathrm{MeCHO}+\mathrm{O}^{\cdot} \rightarrow \mathrm{MeCO}^{\cdot}+{ }^{\cdot} \mathrm{OH}$

$\mathrm{MeCHO}+{ }^{\cdot} \mathrm{OH} \rightarrow \mathrm{MeCO}^{\cdot}+{ }^{\cdot} \mathrm{OH}$

$\mathrm{MeCO}^{*}+\mathrm{O} \rightarrow \mathrm{MeCOO}^{*} \rightarrow \mathrm{Me}^{*}+\mathrm{CO}_{2}$

$\mathrm{MeCO}^{*} \rightarrow \mathrm{Me}^{\cdot}+\mathrm{CO}$

Me: methyl $\left(-\mathrm{CH}_{3}\right)$

Fig. 5. Reaction scheme of atomic oxygen on polymer surface. 


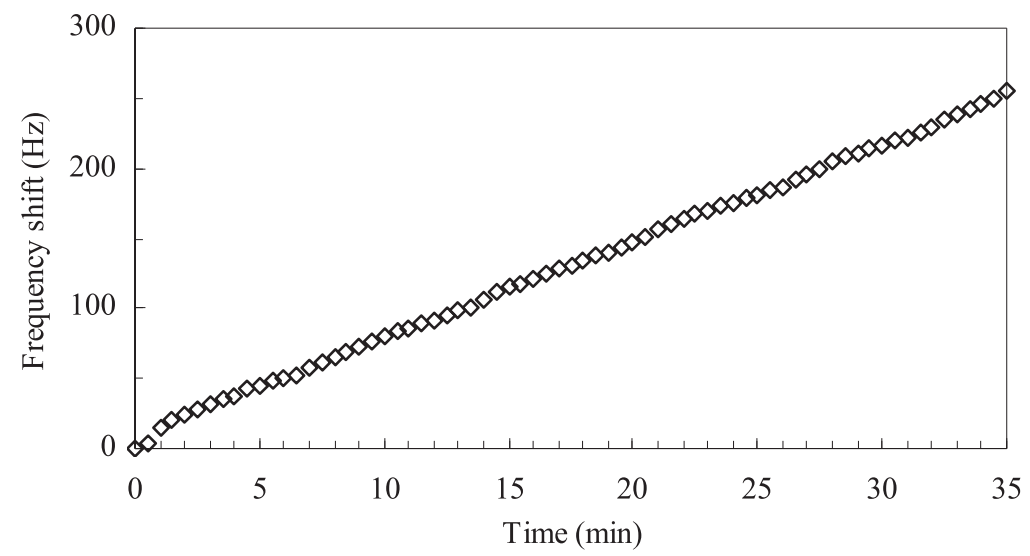

Fig. 6. Characteristic of atomic oxygen sensing using sputter-coated QCM for more than $30 \mathrm{~min}$ (atomic oxygen flux: $6.93 \times 10^{13}$ atoms $/ \mathrm{cm}^{2} / \mathrm{s}$ ).

extended periods, compared with the conventional QCM.

In this study, real-time monitoring of atomic oxygen and its quantification were verified. It is known that atomic oxygen is the predominant species not only in the plasma process but also in the low-pressure mercury ultraviolet lamp and/or xenon excimer lamp processes under atmospheric pressure. ${ }^{(14)}$ Because atomic oxygen has a rate constant that is three orders of magnitude higher than that of ozone, the effect on the surface is noticeable. ${ }^{(15)}$ Therefore, the QCM-based sensor has a great potential for the detection of atomic oxygen generated under UV lamps.

\section{Conclusions}

We investigated a new atomic oxygen sensing method using a QCM with a polymer thin film prepared by RF sputtering. The sputter-coated polymer QCM fully satisfied our demands, because of its sensing characteristics, high sensitivity, and suitability for mass production with excellent reproducibility. Moreover, this study revealed the mechanism of atomic oxygen sensing. This knowledge has led to the adoption of the QCM method for practical industrial applications. Furthermore, from these facts, it is possible to easily identify atomic oxygen species using the frequency shift of the sputter-coated polymer QCM.

\section{References}

1 R. Ono, Y. Yamashita, K. Takezawa and T. Oda: J. Phys. D: Appl. Phys. 38 (2005) 2812.

2 S. Takashima, M. Hori and T. Goto: Appl. Phys. Lett. 75 (1999) 3929.

3 H. Nagai, M. Hiramatsu, M. Hori and T. Goto: Rev. Sci. Instrum. 74 (2003) 3453. 
4 G. Z. Sauerbrey: Z. Phys. 155 (1959) 206.

5 K. Noda, R. Naganawa, Y. Kanekiyo and H. Tao: Chem. Lett. 32 (2003) 1128.

6 H. Matsumoto, M. Matsuoka and K. Noda: Proceedings of the International Conference on Control and Automation Systems 2008 (ICCAS 2008, Seoul, Korea, October 2008) p. 130.

7 M. A. Golub and T. Wydeven: Polym. Degrad. Stab. 22 (1988) 325.

8 A. Uemura, S. Yano, S. Iwamori and K. Noda: J. Mater. Sci. Soc. Jpn. 45 (2008) 143 (in Japanese).

9 H. Matsumoto, M. Matsuoka and K. Noda: Chem. Lett. 34 (2009) 216.

10 S. Iwamori, K. Kezuka and A. Uemura: Mol. Cryst. Liq. Cryst. 471 (2007) 99.

11 H. Kinoshita, M. Tagawa, M. Ueno and N. Ohmae: Trans. Japan Soc. Aero. Space Sci. 41 (1998) 94.

12 K. Yokota, S. Seikyu, M. Tagawa and N. Ohmae: Proceedings of the 9th International Symposium on Material in a Space Environment (Noordwijk, Netherlands, June 2003) p. 265.

13 S. Iwamori, N. Yanagawa, M. Sadamoto, R. Nara and S. Nakahara: in Recent Research Developments in Vacuum Science \& Technology Vol. 4, ed. S. G. Pandalai (Research Signpost, Trivandrum, 2003) p. 49.

14 J. R. Vig: Proc. Electrochem. Soc. 190 (1990) 9.

15 H. Okabe: Photochemistry of Small Molecules, (Wiley-Interscience, New York, 1987) 177. 